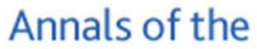 \\ RHEUMATIC DISEASES \\ The Eular Journal
}

\section{Regulatory T Cells in Rheumatoid Arthritis Showed Increased Plasticity toward Th17 but Retained Suppressive Function in Peripheral Blood}

\begin{tabular}{|c|c|}
\hline Journal: & Annals of the Rheumatic Diseases \\
\hline Manuscript ID: & annrheumdis-2013-204228.R1 \\
\hline Article Type: & Extended report \\
\hline Date Submitted by the Author: & $\mathrm{n} / \mathrm{a}$ \\
\hline Complete List of Authors: & $\begin{array}{l}\text { Wang, Tian; Peking University People's Hospital and Capital Medical } \\
\text { University Affiliated Anzhen Hospital, Department of Rheumatology and } \\
\text { Immunology } \\
\text { Sun, Xiaolin; Peking University People's Hospital, Department of } \\
\text { Rheumatology and Immunology } \\
\text { Zhao, Jing; Peking University People's Hospital, Department of } \\
\text { Rheumatology and Immunology } \\
\text { Zhang, Jing; Peking University People's Hospital, Department of } \\
\text { Rheumatology and Immunology } \\
\text { Zhu, Huaqun; Peking University People's Hospital, Department of } \\
\text { Rheumatology and Immunology } \\
\text { Li, Chun; Peking University People's Hospital, Department of Rheumatology } \\
\text { and Immunology } \\
\text { gao, na; Peking Union Medical College Hospital, Peking Union Medical } \\
\text { College and Chinese Academy of Medical Sciences, Department of } \\
\text { Rheumatology } \\
\text { Jia, Yuan; Peking University People's Hospital, Department of } \\
\text { Rheumatology and Immunology } \\
\text { Xu, Dakang; Monash Institute of Medical Research, Monash University } \\
\text { Huang, Fang-Ping; Imperial College Faculty of Medicine, Hammersmith } \\
\text { Hospital, Division of Immunology \& Inflammation (Rheumatology Section), } \\
\text { Department of Medicine } \\
\text { Li, Ningli; Shanghai Jiao Tong University School of Medicine, Shanghai } \\
\text { Institute of Immunology, Institute of Medical Sciences } \\
\text { Lu, Liwei; The University of Hong Kong, Department of Pathology and } \\
\text { Center of Infection and Immunology } \\
\text { Li, Zhanguo; Peking University People's Hospital, Department of } \\
\text { Rheumatology and Immunology }\end{array}$ \\
\hline Keywords: & Rheumatoid Arthritis, T Cells, Cytokines, Inflammation \\
\hline
\end{tabular}

\section{SCHOLARONE \\ Manuscripts}




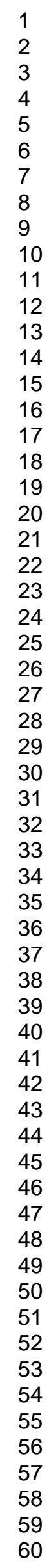

http://mc.manuscriptcentral.com/ard 


\section{Regulatory T Cells in Rheumatoid Arthritis Showed Increased Plasticity toward Th17 but Retained Suppressive Function in Peripheral Blood}

Tian Wang ${ }^{1,2, *}$, MD, Xiaolin Sun ${ }^{1, *}, \mathrm{PhD}$, Jing Zhao ${ }^{1}$, MS, Jing Zhang ${ }^{1}$, MS, Huaqun Zhu ${ }^{1}$, MS, Chun $\mathrm{Li}^{1}, \mathrm{MD}, \mathrm{Na} \mathrm{Gao}^{2}, \mathrm{MD}$, Yuan Jia ${ }^{1}, \mathrm{MD}$, Dakang $\mathrm{Xu}^{4}, \mathrm{PhD}$, Fang-Ping Huang ${ }^{5}, \mathrm{PhD}$, Ningli $\mathrm{Li}^{6}, \mathrm{PhD}$, Liwei $\mathrm{Lu}^{3, \Delta}, \mathrm{PhD}$, Zhan-Guo $\mathrm{Li}^{1,}{ }^{1,}, \mathrm{MD}$, $\mathrm{PhD}$

${ }^{1}$ Department of Rheumatology \& Immunology, Clinical Immunology Center, Peking University People's Hospital, Beijing China; ${ }^{2}$ Department of Rheumatology \& Immunology, Capital Medical University Affiliated Anzhen Hospital, Beijing, China.

${ }^{3}$ Department of Pathology and Center of Infection and Immunology, The University of Hong Kong, Hong Kong, China; ${ }^{4}$ Center for cancer research, Monash Institute of Medical Research, Monash University, Australia; ${ }^{5}$ Division of Immunology \& Inflammation (Rheumatology Section), Department of Medicine, Imperial College Faculty of Medicine, Hammersmith Hospital, London, UK, ${ }^{6}$ Shanghai Institute of Immunology, Institute of Medical Sciences, Shanghai Jiao Tong University School of Medicine.

* Tian Wang and Xiaolin Sun contributed equally to this work.

${ }^{\Delta}$ Names and addresses for correspondence and request for reprints: 
Prof. Zhan-Guo Li,

Department of Rheumatology \& Immunology/Clinical Immunology Center,

People's Hospital, Peking University;

Beijing, China, 100044;

Tel: 86-10-88324172, Fax: 86-10-88378021, E-mail: li99@ bjmu.edu.cn;

or

Prof. Liwei Lu,

Department of Pathology and Center of Infection and Immunology,

The University of Hong Kong, Hong Kong, China.

Email: liweilu@hku.hk

Supported by National Basic Research Program of China (973 program, 2010CB529104), National Natural Science Foundation of China (31000409) and Chinese Medical Association Clinical Medical Research Foundation (12040630363). 


\begin{abstract}
Objective. Regulatory $\mathrm{T}$ cells (Tregs) with the plasticity of producing proinflammatory cytokine IL-17 have been demonstrated under normal and pathogenic conditions. However, it remains unclear whether IL-17-producing Tregs lose their suppressive functions because of their plasticity toward Th17 in autoimmunity. The aim of this study was to investigate IL-17-producing Tregs from patients with rheumatoid arthritis (RA) and characterize their regulatory capacity and clinical significance.
\end{abstract}

Methods. Foxp3 and IL-17 co-expression were evaluated in CD4+ T lymphocytes from RA patients. An in vitro T cell polarization assay was performed to investigate the role of proinflammatory cytokines in IL-17-producing Treg polarization. The suppressive function of IL-17-producing Tregs in RA was assessed by an in vitro suppression assay. The relationship between this Treg subset and clinical features in RA patients was analyzed using Spearman's rank correlation test.

Results. A higher frequency of IL-17-producing Tregs was present in the peripheral blood of RA patients compared with healthy subjects. These cells from peripheral blood showed phenotypic characteristics of both Th17 and Treg cells, and suppressed T cell proliferation in vitro. Tregs in RA synovial fluid lost suppressive function. The Th17 plasticity of Tregs could be induced by IL-6 and IL-23. An increased ratio of this Treg subset was associated with decreased levels of inflammatory markers, including the erythrocyte sedimentation rate and C-reactive protein level, in patients with RA. 
2

3

4

Conclusion. Increased levels of IL-17-producing Tregs were identified in RA patients. This Treg subset with Th17 plasticity in peripheral blood retained suppressive functions and was associated with milder inflammatory conditions, suggesting that this Treg population works as a negative regulator in RA, but in RA synovial site it may be pathogenic.

Key words: plasticity, regulatory T cell, rheumatoid arthritis, Th17 


\section{INTRODUCTION}

IL-17-producing CD4+ T cells (Th17 cells) and Foxp3+ regulatory T cells (Treg cells) have been implicated in the pathogenesis of various chronic autoimmune diseases, including rheumatoid arthritis (RA) [1-4]. Increased Th17 cells mediate autoreactive inflammation and tissue destruction during RA development [5-8], whereas several types of Treg cells maintain tight regulation of T helper (Th) cells for preventing $\mathrm{T}$ cell-mediated autoimmunity and inflammatory damage [9-12]. Recent studies have revealed a link between Treg and Th17 cells. Treg and Th17 cells may develop from the same precursors under distinct cytokine conditions [13], and a subset of IL-17-producing CD4+Foxp3+ Treg cells can be generated upon polarization by cytokines such as IL-6 [14-16].

The observed Th17 plasticity of Treg cells has raised the possibility that the stability of Foxp3+ Treg cells may play a potential pathogenic role at sites of inflammation. Treg cells with the plasticity of producing the proinflammatory cytokine IL-17 have been detected in normal human peripheral blood as well as in inflamed intestinal mucosa in patients with Crohn's disease [16, 18]. However, conflicting data exist regarding the functions of the IL-17-producing Treg population. Previous studies have reported that the induction of IL-17 expression in Treg cells under inflammatory conditions was accompanied by impaired or lost suppressive function [26, 27]. In contrast, Hovhannisyan et al. showed that IL-17-producing Treg cells from patients with Crohn's disease retained suppressive activity despite their increased IL-17 production, although the authors suggested that resident Treg cells under inflammatory conditions may convert to IL-17-producing cells and thus 
potentially contribute to the pathogenesis of the disease [18]. To date, the exact role of Treg plasticity in immunoregulation is unclear, and the presence and clinical significance of the IL-17-producing Treg subset in RA remains unknown.

In the present study, we investigated the CD4+ Foxp3+ IL-17-producing Treg cell in patients with RA. This Treg population exhibited phenotypic characteristics of both Treg and Th17 cells, and retained potent suppressive activity in peripheral blood. However, these Treg cells in RA synovial site lost suppressive activity. The expansion of this Treg population was promoted by the inflammatory cytokines IL-6 and IL-23, which were abnormally elevated in patients with RA. Moreover, the peripheral IL-17-producing Treg subset was associated with decreased RA disease activity, suggesting that these Treg cells are negative regulators in RA peripheral blood, but they might be pathogenic in RA synovial sites..

\section{MATERIALS AND METHODS}

\section{Flow cytometry}

Isolated PBMCs were stained for CD4 expression using a PE-CF594-labeled anti-human-CD4 antibody, for CD127 expression using a PerCP-Cy5.5-labeled anti-human-CD127 antibody, and for CD25 expression using a PE-Cy7-labeled anti-human-CD25 antibody (all antibodies from BD Pharmingen, San Diego, CA, USA). The expression of CTLA-4, IL-10, CD161, and CCR6 was examined using FITC-labeled monoclonal antibodies (BD Pharmingen). To analyze cytokine production, cells were stimulated and incubated for $5 \mathrm{~h}$ with $50 \mathrm{ng} / \mathrm{ml}$ phorbol 
myristate acetate (PMA), $1 \mu \mathrm{g} / \mathrm{ml}$ ionomycin (both from Sigma-Aldrich, Germany), and $1 \mu \mathrm{l} / \mathrm{ml}$ GolgiStop (BD Biosciences, Franklin Lakes, NJ, USA). After stimulation, the cell surface markers CD4, CD25, and CD127 were stained with the fluorescently labeled monoclonal antibodies mentioned above. Next, the cells were fixed and permeabilized for 30 minutes at room temperature at dark with the Foxp3 Fixation/Permeabilization Concentrate and Diluent buffer set (eBioscience). Then the cells were stained with PE-labeled anti-human Foxp3 monoclonal antibody (eBioscience) and Alexa Fluor 647-labeled anti-human-IL-17A antibody or PE-labeled anti-mouse/human ROR $\gamma \mathrm{t}$ antibody (eBioscience). The cells were examined by flow cytometry (BD Aria II flow cytometer), and the data were analyzed using FlowJo v5.7 software (Tree Star, Inc., USA).

Other detailed information about sample preparation, experimental and statistical methods are available in the Supplementary Materials

\section{RESULTS}

\section{IL-17-producing Treg cells were present in the peripheral blood of patients with} RA

To verify the plasticity of Treg cells to produce Th17 under autoimmune inflammatory conditions, IL-17 production and Foxp3 expression in CD4+ T cells were examined using flow cytometry of PBMCs from patients with RA and healthy subjects. IL-17-producing CD4+ Foxp3+ Treg cells were present in patients with RA 
and healthy controls (Figure 1A), and the frequency of IL-17-producing CD4+ Foxp3+ Treg cells was significantly higher in patients with RA than in healthy controls (Figure 1B). Notably, in patients with RA, there is no significant correlation between IL-17-producing Treg cells and CD4+ Foxp3+ Treg cells (Figure 1C), and this IL-17-producing Treg population showed significantly positive correlation with Th17 cells (Figure 1D). We further examined the IL-17 producing Treg cell frequency in matched synovial fluid and peripheral blood from RA patients, and found that the frequency of this Treg subset was significantly elevated in synovial fluid (Figure 1E and Supplementary Figure 1).

A previous study has defined three cell fractions with different functional characteristics in CD4+CD25+ Treg cells with CD45RA and Foxp3 expression [21], Figure 1F upper panel]. Our study showed that more than $60 \%$ of IL-17 producing Treg cells were enriched in CD45RA- Foxp3 3 low "non-Treg" fraction, and around 30\% IL-17 producing Treg cells distributed in CD45RA+ Foxp3 3 low "resting Treg" fraction. Few Tregs in CD45RA- Foxp3 "activating Treg” fraction expressed IL-17 (Figure 1F lower panel).

\section{IL-17-producing Treg cells from the peripheral blood of patients with RA exhibited phenotypic characteristics of both conventional Th17 and Treg cells}

To examine the phenotypic characteristics of the IL-17-producing CD4+ Foxp3+ Treg cells, we used flow cytometry to assess the expression of Foxp3 and surface markers or cytokines known to be associated with either Th17 or Treg cells. The expression levels of the Treg-associated regulatory molecules CTLA-4 and IL-10 
were significantly higher in IL-17-producing CD4+ Foxp3+ Treg cells (MFI: $6.11 \pm$ 0.92 and $4.46 \pm 0.39$, respectively) than in either conventional CD4+ Foxp3+ Treg cells (MFI: $2.77 \pm 0.10$ and $2.69 \pm 0.21$, respectively; $p<0.05$ for each) or Th17 cells (MFI: $3.67 \pm 0.48$ and $3.25 \pm 0.07$, respectively; $p<0.05$ for each), whereas Foxp3 expression was slightly but significantly lower in IL-17-producing CD4+ Foxp3+ Treg cells than in conventional Treg cells (MFI: $68.74 \pm 13.80 v s .77 .88 \pm 16.00, p<$ 0.05) (Figure 2). CCR6 and CD161 are human Th17 cell markers [24], whereas conventional Treg cells express characteristically low levels of CD127 [20]. Similar to Th17 cells, IL-17-producing CD4+ Foxp3+ Treg cells expressed higher levels of CCR6, CD161, and CD127 compared with conventional Treg cells (CCR6 MFI: 3.40 \pm 0.16 vs. $1.86 \pm 0.07, p<0.05$; CD161 MFI: $13.99 \pm 2.98$ vs. $1.86 \pm 0.07, p<0.05$ CD127 MFI: $32.29 \pm 5.68$ vs. $13.48 \pm 2.36, p<0.05$; Figure $2 \mathrm{~A}$ and B). These results show that IL-17-producing CD4+ Foxp3+ Treg cells exhibit phenotypes of both conventional Th17 and Treg cells.

\section{Inflammatory cytokines in RA promoted the induction of IL-17-producing Treg} cells

To characterize the inflammatory milieu contributing to the plasticity of Treg cells in the peripheral blood from patients with RA, we assessed the concentrations of different proinflammatory cytokines in serum samples. Serum levels of IL-6 and IL-23 were significantly elevated in patients with RA compared with healthy controls (IL-23: $32.67 \pm 34.50$ vs. $1.82 \pm 1.51$ pg/ml, $p<0.001$; IL-6: $157.83 \pm 27.07$ vs. 21.97 $\pm 3.52 \mathrm{pg} / \mathrm{ml}, p<0.001$; Figure $3 \mathrm{~A})$. 
Because IL-6 and IL-23 together promote the development and expansion of Th17 cells [9-11], elevated levels of these two cytokines in the sera of patients with RA may also provide the polarizing environment for the expansion of IL-17-producing Treg cells. To test this hypothesis, CD4+CD25+ Treg cells from the peripheral blood of patients with RA or healthy controls were purified by cell sorting and cultured in vitro in the presence of IL-2, IL-1 $\beta$, and anti-CD3 and anti-CD28 antibody coated beads and supplemented with IL-6, IL-23, or both for 5 days. As expected, relatively low frequency of IL-17-producing Foxp3+ Treg cells was detected in cultures without the addition of polarizing cytokines. In the presence of IL-23, there was a slight but not significant increase in the IL-17-producing Treg frequency. However, under the polarizing culture condition supplemented with IL-6 or both IL-6 and IL-23, a significantly increased IL-17-producing Treg cell frequency was observed (Figure 3B and C), implicating the polarizing function of IL-6 and IL-23 and that IL-6 should be a major player in the induction of IL-17-producing Treg cells. However, we did not observe any significant correlation between the serum levels of these polarizing cytokines and the IL-17 producing Treg subset (Supplementary Figure 2). Compared to Tregs from RA patients, Tregs from healthy controls showed similar plasticity toward Th17 under the same polarizing culture conditions (Figure 3B and C), though the IL-17 producing Treg frequencies were modestly lower than those from RA patients without statistical significance. We also examined the expression of Th17-lineage specific transcription factor $\mathrm{ROR} \gamma \mathrm{t}$ in Treg cells cultured in polarizing conditions. The RORgt levels were obviously increased when IL-6 or IL-6 together 
with IL-23 was added to culture (Figure 3D).

\section{CD161 expression defined IL-17-producing Treg subset}

We found that CD161, a surface marker of Th17 precursor cells [24], was dramatically increased on IL-17 producing Treg cells (Figure 2), and it could also be used as the surface marker to characterize IL-17 producing Treg cells (Figure 4A). With CD161 expression, we sorted CD4+CD25+ Treg cells from RA peripheral blood into two subsets: CD4+CD25+CD161+ Treg and CD4+CD25+CD161- Treg (Figure 4A upper panel). Most IL-17 producing Treg cells were enriched in the CD161+ Treg population and few CD161- Treg cells expressed IL-17 (Figure 4A middle panel). Besides IL-17 production, the CD4+CD25+CD161+ Treg cells also expressed significantly higher ROR $\gamma \mathrm{t}$ than the CD161- Treg population (Figure 4A lower panel), which further confirmed that CD4+CD25+CD161+ Treg population was the IL-17 producing Treg subset and processed increased plasticity toward Th17. Similar results were also obtained in Treg cells from synovial fluid of RA patients and peripheral blood of healthy controls (data not shown).

Peripheral IL-17-producing Treg cells from patients with RA retained suppressive function in vitro

We next investigated whether the IL-17-producing Treg cells from patients with RA possessed suppressive function. Although IL-17 producing Treg cells expressed IL-17, we also observed significantly elevated immunosuppressive molecular IL-10 
and CTLA-4 expression in this Treg subset than in conventional Treg cells (Figure 2B), which implicated the possibility that IL-17 producing Treg cells might play a role in immunosuppression.

By CD161 staining, it was feasible to sort and enrich fresh IL-17 producing Treg cells from peripheral blood or synovial fluid. We set up Treg suppression assay with CD4+CD25+CD161+ IL-17 producing Treg cells and CD4+CD25+CD161- Treg cells from peripheral blood of RA patients and healthy controls as well as these two Treg subsets from RA synovial fluid. The CD4+CD25+CD161+ Treg cells and CD4+CD25+CD161- Treg cells from both RA and healthy peripheral blood showed comparable suppression activity on CD4+CD25- responder T-cell (Tresp) proliferation (Figure 4B). In contrast to Tregs from peripheral blood, both CD161+ IL-17 producing Tregs and CD161- Tregs from RA synovial fluid did not inhibit Tresp proliferation (Figure 4B). This result shows that IL-17-producing Treg cells circulating in peripheral blood retained a potent suppressive function to inhibit $\mathrm{T}$ cell proliferation, but the immunosuppressive activity of Treg cells in RA synovial fluid was seriously impaired.

\section{Associations between circulating IL-17-producing Treg cells and RA clinical features}

To determine the clinical relevance of IL-17-producing Treg cells in RA, the percentages of this Treg subset among CD4+ Foxp3+ Treg cells in the peripheral blood were measured in a cohort of 42 patients with RA. The percentages of 
IL-17-producing Treg cells among CD4+ Foxp3+ Treg cells were negatively correlated with ESR $(r=-0.50, p<0.01), \mathrm{CRP}(\mathrm{r}=-0.32, p=0.036)$, and total IgG $(\mathrm{r}=-0.371, p=0.022)$ (Figure $5 \mathrm{~A})$. There were also weak but significant positive correlations between this subset and circulating complement levels $(\mathrm{C} 3, \mathrm{r}=0.33, p=$ 0.048; $\mathrm{C} 4, \mathrm{r}=0.32, p=0.045$; Figure 5A), suggesting that $\mathrm{C} 3$ and $\mathrm{C} 4$ levels would decrease with fewer IL-17-producing Treg cells. No significant correlation was found with patient age, disease duration, anti-CCP antibody, RF-IgM, RF-IgG, total IgA, total IgM, tender joint count, swollen joint count, or DAS28. These correlations indicate that the increased ratio of IL-17-producing Treg cells is associated with milder inflammation in patients with RA, which suggests that the circulating IL-17 producing Treg subset plays a negative regulatory role in the autoimmunity of RA. In addition, the ratio of IL-17-producing Treg cells was significantly lower in CD4+ Treg cells from patients with high RA disease activity (DAS28 > 5.1) than in cells from patients with intermediate or low disease activity (DAS28 $<5.1$; Figure 5B). These results were consistent with our data from in vitro suppressive assays, suggesting that circulating IL-17-producing Treg cells in patients with RA retain their regulatory function in the inflammatory conditions of RA.

\section{DISCUSSION}

Recent studies have reported plasticity in the differentiation of Treg and Th17 cells. Foxp3+ Treg cells can be converted into inflammatory cytokine-producing cells in the specific inflammatory microenvironment and may potentially contribute to 
disease pathogenesis [14-20]. However, the contributions of Treg plasticity to the pathogenesis of autoimmune diseases require further study. In this study, we report for the first time the identification of IL-17-producing CD4+ Foxp3+ Treg cells in patients with RA. The frequency of this IL-17-producing Treg population is significantly increased in peripheral blood and synovial fluid of RA patients compared with that in healthy controls, which suggests that this Treg cell subset is associated with RA pathology.

We evaluated the distribution of IL-17 producing Treg in CD4+CD25+ Treg subpopulations defined with different CD45RA and Foxp3 expression by Sakaguchi group [21]. The majority of IL-17 producing Treg cells (over 60\%) were from the CD45RA- Foxp3 $3^{\text {low }}$ "non-Treg" fraction, which was considered transiently and unstably expressing Foxp3 and was shown to lack suppressive activity and had Th17 cell potential [21], while relatively fewer IL-17 producing Treg cells were also distributed in the CD45RA+ Foxp3 ${ }^{\text {low }}$ "resting Treg" fraction (around 30\%) and CD45RA- Foxp3 "activating Treg" fraction (less than 10\%), both of which stably expressed Foxp3 and were suppressive [21]. This result clearly revealed that IL-17 producing Treg cells derived from different Treg lineages, and Treg plasticity toward Th17 existed in all these subpopulations.

We observed that circulating IL-17-producing Foxp3+ Treg cells in peripheral blood exhibited phenotypes of Th17 cells, including IL-17 secretion and elevated expression of RORyt, CD161 and CCR6. CD161 has been identified to be an important Th17 molecular marker. Nearly all Th17 cells were contained in CD161+ 
population and originated from CD161+ naïve T cells [24]. In this study, we also identified CD161 as the key surface marker to characterize IL-17 producing Treg cells. The CD4+CD25+CD161+ Treg subset enriched nearly all of the IL-17 producing Treg cells and the ROR $\gamma$ t expression was significantly increased in this subset, which indicated that the CD161 specifically defined the Treg subset producing IL-17 and possessing high plasticity toward Th17.

Besides the Th17 lineage markers, the IL-17 producing Treg cells from peripheral blood of RA patients continued to express the anti-inflammatory factors IL-10 and CTLA-4 and to retain functional suppressive activity in vitro. In addition, we found a significant negative correlation between this Treg subset in peripheral blood and RA inflammation markers (ESR, CRP, total IgG and C3/C4 decreasement), which indicated that inflammation and autoimmune responses might be milder with increased peripheral IL-17-producing Treg cells in patients with RA. This clinical relevance is consistent with the potent suppressive function of RA peripheral IL-17-producing Treg cells. Our data are in agreement with the recent finding that IL-17-positive Treg cell clones from the inflamed intestinal mucosa of patients with Crohn's disease remained suppressive in vitro [18], and our results support those of an in vitro investigation by Walter et al., which showed that the IL-17-producing Treg cells induced by RA-related cytokine-activated monocytes in vitro exhibited enhanced inflammatory cytokine expression and suppressive function [25]. Together with findings from different experimental systems, our study suggests that an increased proinflammatory cytokine IL-17-expressing Treg population in peripheral blood acts 
as a negative regulator in RA development by maintaining or enhancing suppressive capacity.

The IL-17-producing Treg subset exhibits phenotypic characteristics of both Treg and Th17 cells. As a result of the overproduction of proinflammatory cytokines in RA, it is possible that Treg cells may be skewed toward IL-17-producing Foxp3+ Treg cells in the presence of inflammatory mediators, thereby favoring Th17 differentiation in patients with RA. Although these cells in peripheral blood could play a regulatory role in RA, we cannot exclude the possibility that some cells in some circumstances may functionally shift from regulatory $\mathrm{T}$ cells to inflammatory effectors expressing IL-17 but lost suppressive functions. In this study, we observed increased frequency of IL-17 producing Tregs in RA synovial fluid, but there was a seriously loss of Treg suppressive activity in RA synovial fluid (Figure 1E and 4B). It is likely that in peripheral blood, the pathogenic function of IL-17-producing Foxp3+ Treg cells is limited because of their relatively low frequency and retaining suppressive functions. However, in RA synovial tissues, proinflammatory cytokines such as TNF $\alpha$, IL-1, IL-6, etc. dramatically increased than in peripheral blood [28, 29]. IL-1 or IL-6 increase might promote the Treg plasticity toward Th17, leading to elevated IL-17 producing Treg frequency in synovial fluid. Meanwhile, TNF $\alpha$ overexpression disabled Treg suppression in synovial fluid by dephosphorylating Foxp3 [30]. Thus, in RA synovial sites, IL-17 producing Treg cells losing suppressive function may act more like Th17 cells, which will promote the joint inflammation due to heavily impaired suppressive function as well as their IL-17 production and increased 
frequency. The IL-17-producing Treg subset may remain suppressive in RA peripheral blood but contribute to autoimmunity in synovial sites.

We detected significantly elevated levels of IL-6 and IL-23 in the peripheral blood of patients with RA compared with healthy controls. Importantly, both IL-6 and IL-23 have been recognized as key contributors to Th17 cell differentiation. IL-6 is engaged in polarizing Th17 cell differentiation, whereas IL-23 is critical for Th17 cell stabilization and expansion $[20,22,23]$. Therefore, there may be a Th17 cell polarizing environment that consists of Th17-related proinflammatory cytokines in the periphery blood of patients with RA. In the present study, IL-6 alone or IL-6 and IL-23 potently promoted the production of IL-17 by purified CD4+ CD25+ Treg cells from both RA and HC peripheral blood in vitro, suggesting that IL-17-producing Treg cells may be differentiated from Foxp3+ Treg cells in RA inflammatory conditions. Treg cells from healthy controls and RA patients showed similar plasticity toward Th17 (Figure 3C), which suggested that there might be no significant intrinsic differences in Treg cell sensitivity to polarizing cytokines between RA patients and healthy controls. The higher RA peripheral IL-17 producing Treg frequency might be attributed to the microenvironment with significantly elevated polarizing cytokines in RA peripheral blood.

In conclusion, our data identified increased IL-17-producing regulatory T cells in the presence of elevated IL-6 and IL-23 levels in patients with RA, providing evidence that Treg cells with the plasticity of IL-17 expression in autoimmune conditions could retain their suppressive capacity. The peripheral Treg cell population 
with increased Th17 plasticity may still play a role as negative regulator in RA autoimmunity, but they lose suppressive activity in synovial sites and may become pathogenic. Future investigations regarding this cell population and the development of Treg/Th17 plasticity will provide a better understanding of the immunopathogenesis of RA.

\section{Competing interests: None.}

Contributorship: Liwei Lu and Zhanguo Li designed the research. Tian Wang and Xiaolin Sun performed the research and analysed the data. Tian Wang, Xiaolin Sun, Liwei Lu and Zhanguo Li wrote the manuscript. Jing Zhao, Jing Zhang, Huaqun Zhu, Chun Li, Na Gao, Yuan Jia, Dakang Xu, Fang-Ping Huang and Ningli Li helped with sample collection, data analysis and manuscript writing.

\section{ACKNOWLEDGMENTS}

This work was supported by National Basic Research Program of China (973 program, 2010CB529104), National Natural Science Foundation of China (31000409), Major International Joint Research Project of NSFC (No:81120108020), Program of International Science \& Technology Cooperation of MOST (No.2010DFB34000) and Chinese Medical Association Clinical Medicine Research Foundation (12040630363). 


\section{REFERENCES}

1. Chang SH, Reynolds JM, Pappu BP, et al. Interleukin-17C promotes Th17 cell responses and autoimmune disease via interleukin-17 receptor E. Immunity. 2011;35(4):611-621.

2. Qian Y, Liu C, Hartupee J, et al. The adaptor Act1 is required for interleukin 17-dependent signaling associated with autoimmune and inflammatory disease. Nat Immunol. 2007;8(3):247-256.

3. Chen $\mathrm{G}, \mathrm{Li} \mathrm{N}$, Zhang $\mathrm{D}$, et al. Immunological profile and regulatory mechanism induced by vaccination with selected synovial $\mathrm{T}$ cells in rheumatoid arthritis. Arthritis Rheum. 2007;56:453-463.

4. Zhang R, Huynh A, Whitcher G, et al. An obligate cell-intrinsic function for CD28 in Tregs. J Clin Invest. 2013;123(2):580-593.

5. Lin J, Zhou Z, Huo R, et al. Cyr61 induces IL-6 production by fibroblast-like synoviocytes promoting Th17 differentiation in rheumatoid arthritis. J Immunol. 2012;188(11):5776-5784.

6. Zhang Q, Wu J, Cao Q, et al. A critical role of Cyr61 in interleukin-17-dependent proliferation of fibroblast-like synoviocytes in rheumatoid arthritis. Arthritis Rheum. 2009;60(12):3602-3612.

7. Harrington LE, Hatton RD, Mangan PR, et al. Interleukin 17-producing CD4+ effector T cells develop via a lineage distinct from the T helper type 1 and 2 lineages. Nat Immunol. 2005;6(11):1123-1132.

8. Park JS, Lim MA, Cho ML, et al. p53 controls autoimmune arthritis via the STAT3/STAT5-mediated regulation of the Th17-Treg balance. Arthritis Rheum. 2012 Dec 28. doi: 10.1002/art.37841. [Epub ahead of print]

9. Chabaud M, Fossiez F, Taupin JL, et al. Enhancing effect of IL-17 on IL-1-induced IL-6 and leukemia inhibitory factor production by rheumatoid arthritis synoviocytes and its regulation by Th2 cytokines. J Immunol. 1998;161(1):409-414.

10. Yagi H, Nomura $\mathrm{T}$, Nakamura $\mathrm{K}$, et al. Crucial role of FOXP3 in the 
development and function of human $\mathrm{CD} 25+\mathrm{CD} 4+$ regulatory $\mathrm{T}$ cells. Int Immunol. 2004;16(11):1643-1656.

11. Papiernik M, de Moraes ML, Pontoux C, et al. Regulatory CD4 T cells: expression of IL-2R alpha chain, resistance to clonal deletion and IL-2 dependency. Int Immunol. 1998;10(4):371-378.

12. Dieckmann $\mathrm{D}$, Plottner $\mathrm{H}$, Berchtold $\mathrm{S}$, et al. Ex vivo isolation and characterization of $\mathrm{CD} 4(+) \mathrm{CD} 25(+) \mathrm{T}$ cells with regulatory properties from human blood. J Exp Med. 2001;193(11):1303-1310.

13. Bettelli E, Carrier Y, Gao W, et al. Reciprocal developmental pathways for the generation of pathogenic effector TH17 and regulatory T cells. Nature $2006 ; 441: 235-238$.

14. Yang XO, Nurieva R, Martinez GJ, et al. Molecular antagonism and plasticity of regulatory and inflammatory T cells programs. Immunity 2008;29:44-56.

15. Zhou L, Lopes JE, Chong MM, et al. TGF- $\beta$-induced FoxP3 inhibits Th17 cell differentiation by antagonizing ROR $\gamma \mathrm{t}$ function. Nature 2008;453:236-240.

16. Koenen HJ, Smeets RL, Vink PM, et al. Human CD25high FoxP3+ regulatory T cells differentiate into IL-17-producing cells. Blood 2008;112:2340-52.

17. Dominguez-Villar M, Baecher-Allan CM, Hafler DA. Identification of $\mathrm{T}$ helper type 1-like, Foxp3+ regulatory $\mathrm{T}$ cells in human autoimmune disease. Nat Med. 2011;17:673-675.

18. Hovhannisyan Z, Treatman J, Littman DR, et al. Characterization of IL-17 producing regulatory $\mathrm{T}$ cells in inflamed intestinal mucosa from patients with inflammatory bowel diseases. Gastroenterology 2011;140:957-965.

19. Prevoo ML, van't Hof MA, Kuper HH, et al. Modified disease activity scores that include twenty-eight-joint counts. Development and validation in a prospective longitudinal study of patients with rheumatoid arthritis. Arthritis Rheum. 1995;38:44-48.

20. Abdulahad WH, Boots AMH, Kallenberg CGM. FoxP3+ CD4+ T cells in systemic autoimmune diseases: the delicate balance between true regulatory $\mathrm{T}$ cells and effector Th-17 cells. Rheumatology 2011;50:646-656. 
21. Miyara M, Yoshioka Y, Kitoh A, et al. Functional delineation and differentiation dynamics of human CD4+ $\mathrm{T}$ cells expressing the Foxp3 transcription factor. Immunity. 2009; 30(6):899-911.

22. Nishihara M, Ogura H, Ueda N, et al. IL-6-gp130-STAT3 in T cells directs the development of IL-17+ Th with a minimum effect on that of Treg in the steady state. Int Immunol. 2007;19(6):695-702.

23. McGeachy MJ, Chen Y, Tato CM, et al. The interleukin 23 receptor is essential for the terminal differentiation of interleukin 17-producing effector $\mathrm{T}$ helper cells in vivo. Nat Immunol. 2009;10(3):314-324.

24. Cosmi L, Palma RD, Santarlasci V, et al. Human interleukin 17-producing cells originate from a CD161+CD4+ $\mathrm{T}$ cell precursor. $\mathrm{J}$ Exp Med. 2008;205:1903-1916.

25. Walter GJ, Evans HG, Menon B, et al. Interaction with activated monocytes enhances cytokine expression and suppressive activity of human CD4+CD45RO+CD25+CD127low regulatory $\mathrm{T}$ cells. Arthritis Rheum. 2013;65:627-638.

26. Beriou G, Costantino CM, Ashley CW, et al. IL-17-producing human peripheral regulatory $\mathrm{T}$ cells retain suppressive function. Blood 2009;113:4240-4249.

27. Valmori D, Raffin C, Raimbaud I, et al. Human ROR $\gamma \mathrm{t}+\mathrm{TH} 17$ cells preferentially differentiate from naive FOXP3+Treg in the presence of lineage-specific polarizing factors. Proc Natl Acad Sci USA 2010;107:19402-19407.

28. Petrovic-Rackov L, Pejnovic N. Clinical significance of IL-18, IL-15, IL-12 and TNF-alpha measurement in rheumatoid arthritis. Clin Rheumatol. 2006 Jul;25(4):448-52.

29. Boissier MC, Semerano L, Challal S, Saidenberg-Kermanac'h N, Falgarone G. Rheumatoid arthritis: from autoimmunity to synovitis and joint destruction. $\mathbf{J}$ Autoimmun. 2012 Sep;39(3):222-8.

30. Nie H, Zheng Y, Li R,et al. Phosphorylation of FOXP3 controls regulatory T 21 
1

2

3

4

5

6

7

8

9

10

11

12

13

14

15

16

17

18

19

20

21

22

23

24

25

26

27

28

29

30

31

32

33

34

35

36

37

38

39

40

41

42

43

44

45

46

47

48

49

50

51

52

53

54

55

56

57

58

59

60 cell function and is inhibited by TNF- $\alpha$ in rheumatoid arthritis. Nat Med. 2013

Mar;19(3):322-8. 


\section{FIGURE LEGENDS}

Figure 1. IL-17-producing Treg cells in peripheral blood from patients with RA and healthy controls. The expression of Foxp3 and IL-17 in CD4+ T cells from patients with RA and healthy controls was evaluated by intracellular cytokine staining and flow cytometry. A. Representative flow cytometric profile showing IL-17 expression in CD4+ Foxp3+ Treg cells in patients with RA and healthy controls. B. Four independent experiments showed that the percentage of IL-17-producing Treg cells in CD4+ Foxp3+Treg cells was significantly higher in patients with RA $(n=12)$ than in healthy control subjects $(n=7)$ * $\mathrm{p}<0.05$. RA: patients with RA; HC: healthy controls. C. There is no significant correlation between IL-17-producing Treg cells and CD4+ Foxp3+ Treg cells in patients with RA. D. The IL-17-producing Treg cells were positively correlated with Th17 cells in patients with RA. E. there are higher frequency of IL-17-producing Tregs in RA synovial fluid than in matched peripheral blood (n=5); SF: synovial fluid; PB: peripheral blood. F. Distribution of IL-17 producing Treg cells in Treg fractions defined by CD45RA and Foxp3 expression. Numbers on the representative flow cytometry graph indicate IL-17 producing Treg numbers in that Treg fraction, percentages in round brackets indicate the percentages of IL-17 producing Tregs of that fraction in total IL-17 producing Tregs of the representative sample. Fr: Fraction; Fr I: CD45RA+Foxp3low resting Treg cells; Fr II: CD45RA-Foxp3hi activating Treg cells; Fr III: CD45RA-Foxp3low “non-Treg” cells. 
Figure 2. IL-17-producing Treg cells display phenotypic similarities to both Th17 and Treg cells. Surface expression of CTLA-4, CD161, CCR6, and CD127, and intracellular expression of Foxp3 and IL-10 were measured in IL-17-producing CD4+ Foxp3+ Treg cells. The CD4+ Foxp3+ Treg cells and CD4+IL17+ Th17 cells were examined by flow cytometry $5 \mathrm{~h}$ after stimulation with phorbol myristate acetate (PMA)/ionomycin. The expression levels of the surface markers or cytokines were quantified as mean fluorescence intensity (MFI). Data are representative of at least five independent experiments $(\mathrm{n}=5 ; * p<0.05)$. A. Representative overlayed flow cytometric histograms showing expression of CTLA-4, CD161, CCR6, CD127, Foxp3 and IL-10 in CD4+ Foxp3+ Treg cells, IL-17-producing Treg cells and CD4+IL17+ Th17 cells; red histogram, CD4+ Foxp3+ Treg cells; green histogram, IL-17 producing Treg cells; blue histogram, Th17 cells; B. The expression levels of the molecular measured above were quantified as mean fluorescence intensity (MFI). 
Figure 3. Induction of peripheral CD4+ regulatory $\mathrm{T}$ cells into IL-17-producing CD4+Treg cells under RA inflammatory conditions. IL-6 and IL-23 levels in sera from patients with RA and healthy controls were quantified by enzyme-linked immunosorbent assay (ELISA). CD4+ T cells were stimulated with either IL-6 or IL-23, or both in vitro in the presence of anti-CD3 and anti-CD28 mAb-coated beads, IL-2 and IL-1 $\beta$. The expression of Foxp3 and IL-17 was determined by intracellular cytokine staining and flow cytometry. A. IL-6 and IL-23 concentrations in sera of 39 patients with RA (RA) and 27 healthy controls (HC) were assessed by ELISA ( ${ }^{*} p<$ 0.01). B. IL-17 and Foxp3 expression levels in CD4+CD25+ Treg cells from RA patients and healthy controls were examined in the presence or absence of IL-6 or IL-23 in vitro with anti-CD3 and anti-CD28 mAb-coated beads, IL-2 and IL-1 $\beta$. Data are representative of three independent experiments. C. The frequencies of IL-17-producing Treg cells from RA patients and healthy controls were measured in the presence or absence of IL-6 or IL-23 in vitro. Data are from three independent experiments $(* p<0.05)$. D. Comparison of ROR $\gamma \mathrm{t}$ expression in CD4+CD25+ Treg cells in the presence or absence of IL-6 or IL-23 in vitro. Data are representative of two independent experiments. 
Figure 4. IL-17 producing Treg cells were enriched in the CD4+CD25+CD161+ Treg subset. CD4+ CD25- effector T (Tresp) cells stained with CFSE were cultured alone or with IL-17 producing CD4+CD25+CD161+ Treg cells or CD4+CD25+CD161Treg cells with rare IL-17 production. Tresp cells were stimulated with anti-CD3 and anti-CD28 mAb coated beads, and their proliferation in 5 days was determined by flow cytometry. A. CD4+CD25+ Treg cells could be isolated into CD4+CD25+CD161+ subset and CD4+CD25+CD161- subset. IL-17 producing Treg cells were enriched in the CD4+CD25+CD161+ Treg subset. RORgt expression was significantly elevated in CD4+CD25+CD161+ Treg cells $\left({ }^{*} p<0.05\right)$. B. both of the CD4+CD25+CD161+ and CD4+CD25+CD161- Treg subsets from peripheral blood of RA patients and healthy controls showed comparable suppression activity on CD4+CD25- Tresp cell proliferation, while the suppressive activity of CD4+CD25+CD161+ and CD4+CD25+CD161- Treg subsets from RA synovial fluid was dramatically impaired. Data are representative of 3-5 independent experiments $\left({ }^{*} p<0.05\right)$. RA: patients with RA; HC: healthy controls; Tresp: responder T cell. 
Figure 5. Negative correlation of IL-17-producing Treg cells with RA disease activity. The correlations between the percentage of IL-17-producing CD4+ Foxp3+ Treg cells in circulating CD4+ Foxp3+ Treg cells and clinical features of patients with RA ( $\mathrm{n}=$ 42) were analyzed by Spearman's rank correlation test. RA disease activity was evaluated by the 28-joint count disease activity score (DAS28), and the IL-17-producing CD4+ Foxp3+ Treg cell frequencies were compared between patients with high RA disease activity and patients with intermediate and low disease activity. A. The ratio of IL-17-producing CD4+ Foxp3+ Treg cells in circulating CD4+ Foxp3+ Treg cells in patients with RA was negatively correlated with the inflammation markers ESR, CRP, and total IgG, and was positively correlated with C3 and C4 levels. B. Patients with higher circulating IL-17-producing Treg cell ratios had lower RA disease activity. ${ }^{*} p<0.05$. 
Figure 1

B

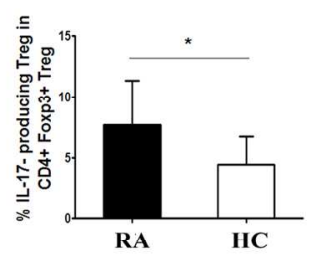

C

D

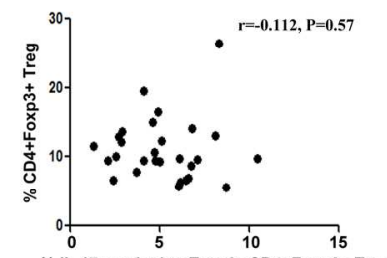

E

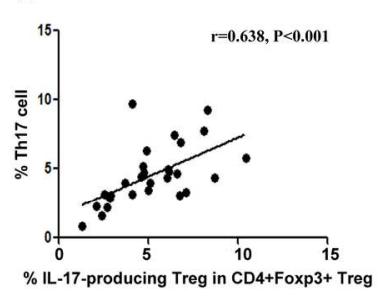

F
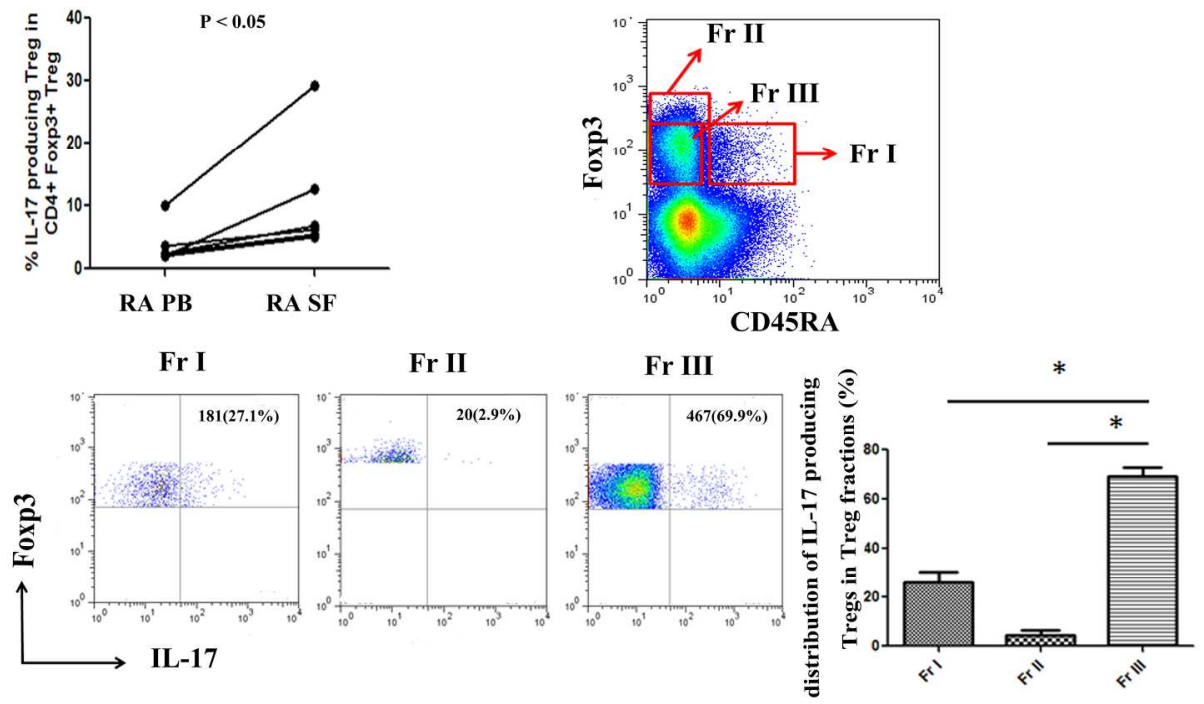

Figure 1. IL-17-producing Treg cells in peripheral blood from patients with RA and healthy controls. The expression of Foxp3 and IL-17 in CD4+ T cells from patients with RA and healthy controls was evaluated by intracellular cytokine staining and flow cytometry. A. Representative flow cytometric profile showing IL-17 expression in CD4+ Foxp3+ Treg cells in patients with RA and healthy controls. B. Four independent experiments showed that the percentage of IL-17-producing Treg cells in CD4+ Foxp3+Treg cells was significantly higher in patients with RA $(n=12)$ than in healthy control subjects $(n=7) * *<0.05$. RA: patients with RA; HC: healthy controls. C. There is no significant correlation between IL-17-producing Treg cells and CD4+ Foxp3+ Treg cells in patients with RA. D. The IL-17-producing Treg cells were positively correlated with Th17 cells in patients with RA. E. there are higher frequency of IL-17-producing Tregs in RA synovial fluid than in matched peripheral blood $(n=5)$; SF: synovial fluid; PB: peripheral blood. $F$.

Distribution of IL-17 producing Treg cells in Treg fractions defined by CD45RA and Foxp3 expression. Numbers on the representative flow cytometry graph indicate IL-17 producing Treg numbers in that Treg fraction, percentages in round brackets indicate the percentages of IL-17 producing Tregs of that fraction in 
total IL-17 producing Tregs of the representative sample. Fr: Fraction; Fr I: CD45RA+Foxp3low resting Treg cells; Fr II: CD45RA-Foxp3hi activating Treg cells; Fr III: CD45RA-Foxp3low "non-Treg" cells. $199 \times 249 \mathrm{~mm}(300 \times 300 \mathrm{DPI})$ 

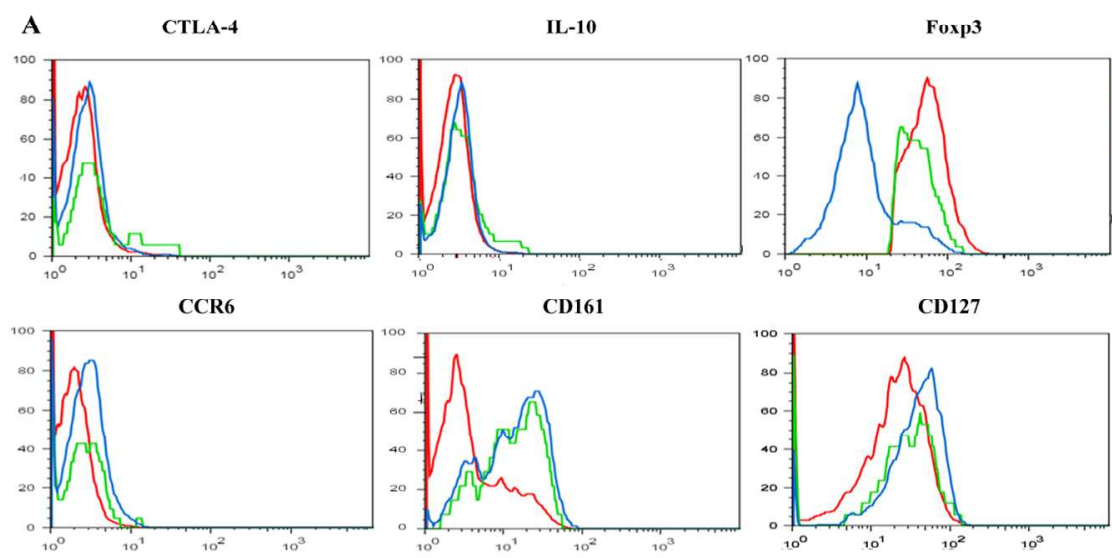

B

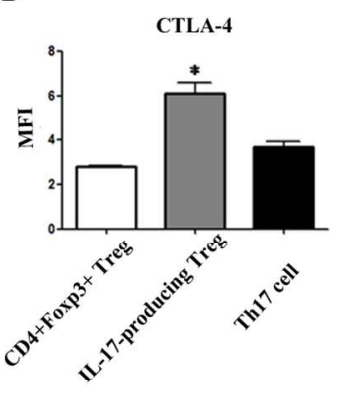

CCR6
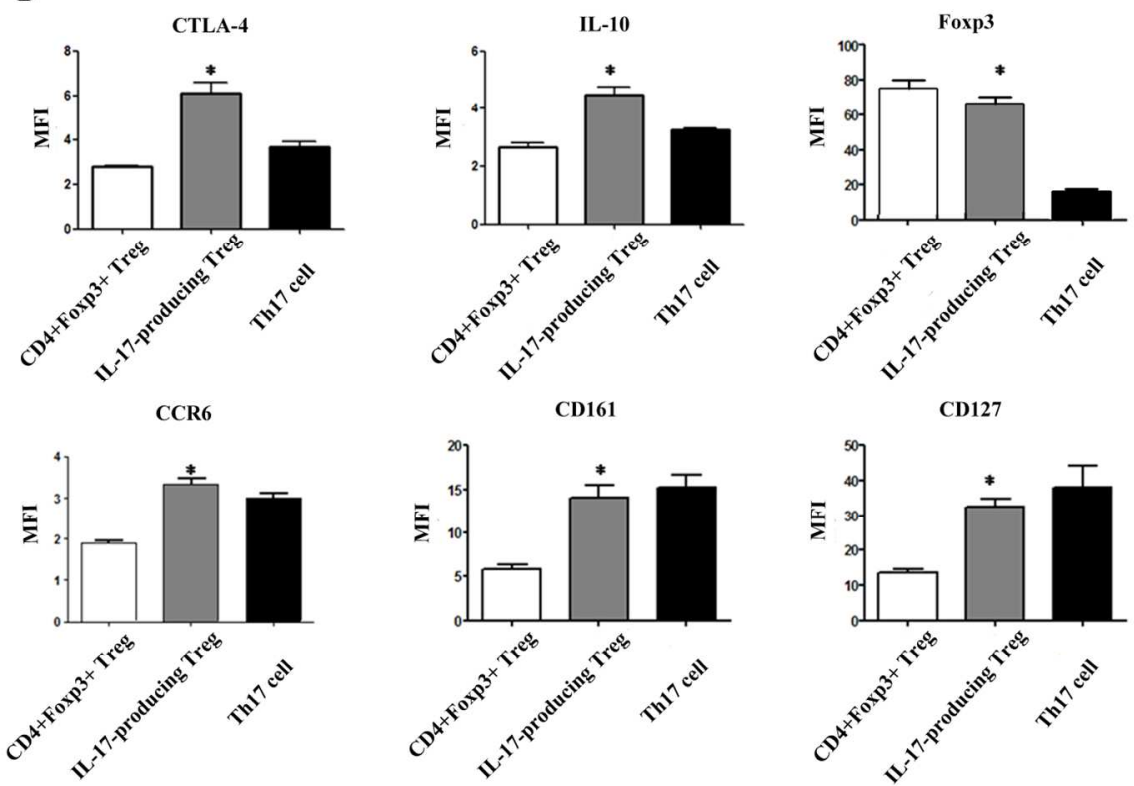

CD161
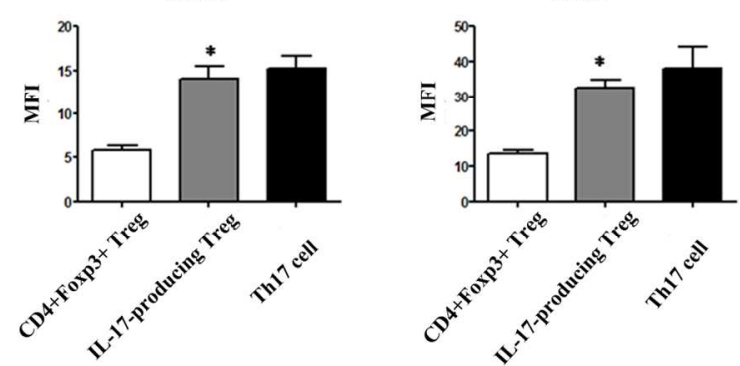

Figure 2. IL-17-producing Treg cells display phenotypic similarities to both Th17 and Treg cells. Surface expression of CTLA-4, CD161, CCR6, and CD127, and intracellular expression of Foxp3 and IL-10 were measured in IL-17-producing CD4+ Foxp3+ Treg cells. The CD4+ Foxp3+ Treg cells and CD4+IL17+ Th17 cells were examined by flow cytometry $5 \mathrm{~h}$ after stimulation with phorbol myristate acetate

(PMA)/ionomycin. The expression levels of the surface markers or cytokines were quantified as mean fluorescence intensity (MFI). Data are representative of at least five independent experiments $(n=5 ; * p<$ 0.05). A. Representative overlayed flow cytometric histograms showing expression of CTLA-4, CD161, CCR6, CD127, Foxp3 and IL-10 in CD4+ Foxp3+ Treg cells, IL-17-producing Treg cells and CD4+IL17+ Th17 cells; red histogram, CD4+ Foxp3+ Treg cells; green histogram, IL-17 producing Treg cells; blue histogram, Th17

cells; $B$. The expression levels of the molecular measured above were quantified as mean fluorescence intensity (MFI).

\section{$129 \times 150 \mathrm{~mm}(300 \times 300 \mathrm{DPI})$}


Figure 3

\section{A}

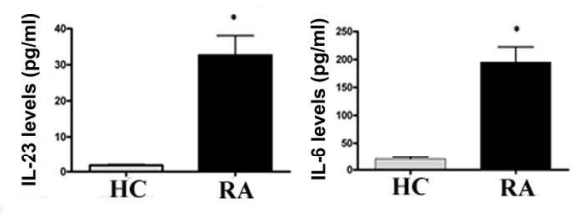

B

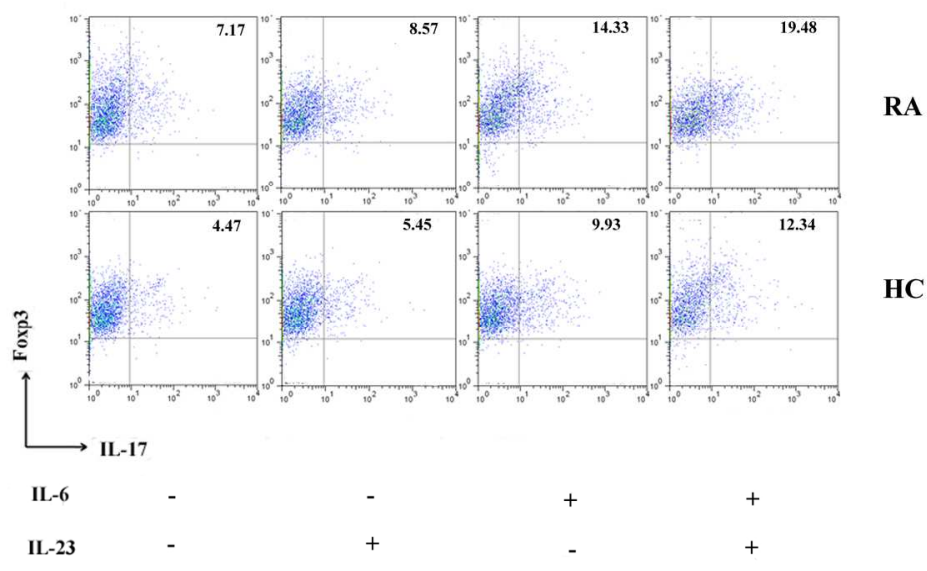

C

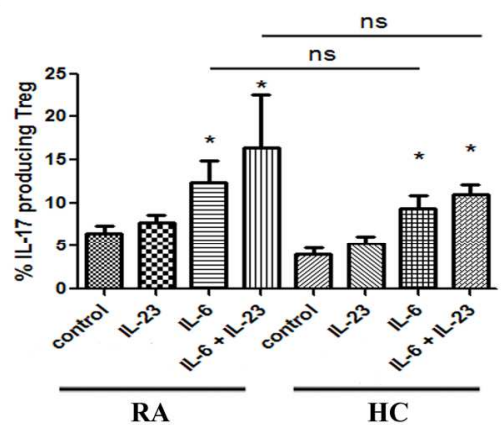

D

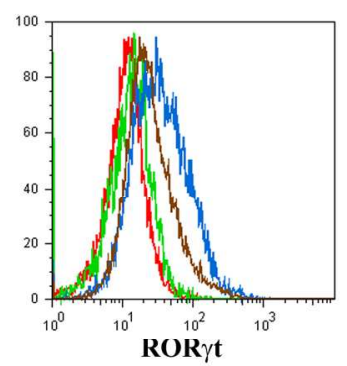

Figure 3. Induction of peripheral CD4+ regulatory T cells into IL-17-producing CD4+Treg cells under RA inflammatory conditions. IL- 6 and IL-23 levels in sera from patients with RA and healthy controls were quantified by enzyme-linked immunosorbent assay (ELISA). CD4+ T cells were stimulated with either IL- 6 or IL-23, or both in vitro in the presence of anti-CD3 and anti-CD28 mAb-coated beads, IL-2 and IL-1 $\beta$. The expression of Foxp3 and IL-17 was determined by intracellular cytokine staining and flow cytometry. A. IL- 6 and IL-23 concentrations in sera of 39 patients with RA (RA) and 27 healthy controls (HC) were assessed by ELISA $(* p<0.01)$. B. IL-17 and Foxp3 expression levels in CD4+CD25+ Treg cells from RA patients and healthy controls were examined in the presence or absence of IL-6 or IL-23 in vitro with anti-CD3 and antiCD28 mAb-coated beads, IL-2 and IL-1 $\beta$. Data are representative of three independent experiments. C. The frequencies of IL-17-producing Treg cells from RA patients and healthy controls were measured in the presence or absence of IL- 6 or IL-23 in vitro. Data are from three independent experiments $(* p<0.05)$. D. Comparison of RORyt expression in CD4+CD25+ Treg cells in the presence or absence of IL-6 or IL-23 in vitro. Data are representative of two independent experiments. 


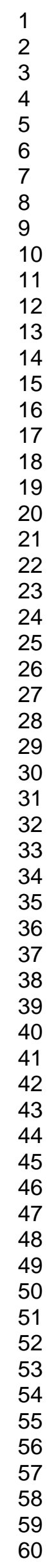

$160 \times 199 \mathrm{~mm}(300 \times 300 \mathrm{DPI})$

http://mc.manuscriptcentral.com/ard 
Figure 4. IL-17 producing Treg cells were enriched in the CD4+CD25+CD161+ Treg subset. CD4+ CD25effector T (Tresp) cells stained with CFSE were cultured alone or with IL-17 producing CD4+CD25+CD161+ Treg cells or CD4+CD25+CD161- Treg cells with rare IL-17 production. Tresp cells were stimulated with anti-CD3 and anti-CD28 mAb coated beads, and their proliferation in 5 days was determined by flow cytometry. A. CD4+CD25+ Treg cells could be isolated into CD4+CD25+CD161+ subset and CD4+CD25+CD161- subset. IL-17 producing Treg cells were enriched in the CD4+CD25+CD161+ Treg subset. RORgt expression was significantly elevated in CD4+CD25+CD161+ Treg cells $(* p<0.05)$. B. both of the CD4+CD25+CD161+ and CD4+CD25+CD161- Treg subsets from peripheral blood of RA patients and healthy controls showed comparable suppression activity on CD4+CD25- Tresp cell proliferation, while the suppressive activity of $C D 4+C D 25+C D 161+$ and $C D 4+C D 25+C D 161-$ Treg subsets from RA synovial fluid was dramatically impaired. Data are representative of 3-5 independent experiments $(* p<0.05)$. RA: patients with RA; HC: healthy controls; Tresp: responder T cell. 


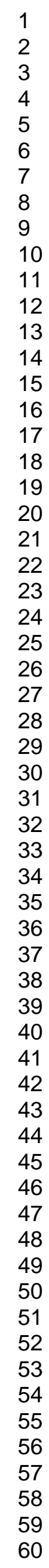

$199 \times 260 \mathrm{~mm}(300 \times 300 \mathrm{DPI})$

http://mc.manuscriptcentral.com/ard 


\section{Figure 5}

A
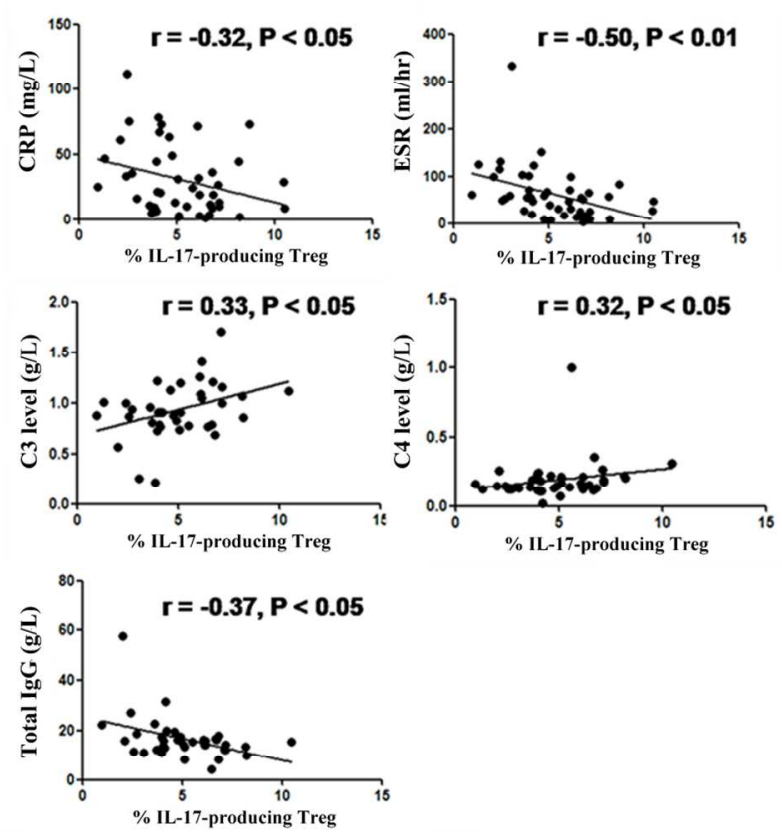

B

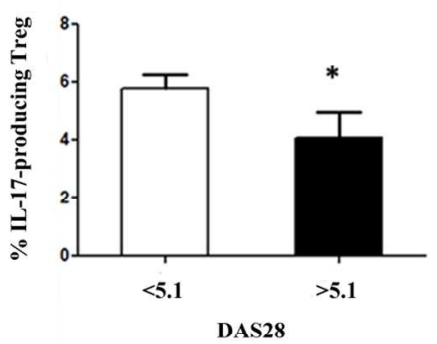

Figure 5. Negative correlation of IL-17-producing Treg cells with RA disease activity. The correlations between the percentage of IL-17-producing CD4+ Foxp3+ Treg cells in circulating CD4+ Foxp3+ Treg cells and clinical features of patients with RA $(n=42)$ were analyzed by Spearman's rank correlation test. RA disease activity was evaluated by the 28-joint count disease activity score (DAS28), and the IL-17producing CD4+ Foxp3+ Treg cell frequencies were compared between patients with high RA disease activity and patients with intermediate and low disease activity. A. The ratio of IL-17-producing CD4+ Foxp3+ Treg cells in circulating CD4+ Foxp3+ Treg cells in patients with RA was negatively correlated with the inflammation markers ESR, CRP, and total IgG, and was positively correlated with C3 and C4 levels. B. Patients with higher circulating IL-17-producing Treg cell ratios had lower RA disease activity. $* p<0.05$. $119 \times 98 \mathrm{~mm}(300 \times 300$ DPI $)$ 


\section{SUPPLEMENTARY MATERIALS}

\section{Supplementary materials and methods}

\section{Patients and samples}

Peripheral blood samples were collected from 42 patients with RA (36 females and 6 males) and 27 healthy donors (16 females and 11 males) at Peking University People's Hospital in Beijing, China. The mean ages of the patients and healthy controls were 53 (range, 27-68) and 49 (range, 25-63) years, respectively. The disease activity score based on 28 joints (DAS28) was calculated to evaluate RA disease activity. All patients met the American College of Rheumatology (ACR) 1987 revised criteria for RA classification and had active arthritis at the time of sampling (DAS28 $\geq 3.2$ ). Patients with RA included in this study were taking methotrexate alone or methotrexate and one nonsteroidal anti-inflammatory drug at the time of sampling, and their mean DAS28 was 4.58 (range, 1.63-7.11). This study was approved by the Ethics Committee of Peking University People's Hospital.

All blood samples were processed within $1 \mathrm{~h}$ of collection. Peripheral blood mononuclear cells (PBMCs) were isolated by Ficoll-Hypaque density gradient centrifugation.

\section{Treg cell polarization assay}

Purified CD4+CD25+ Treg cells were cultured in TexMACS GMP medium (Miltenyi Biotec Inc., USA), 10 IU/ml IL-2, 10ng/ml IL-1 $\beta$ (PeproTech, USA), and anti-CD3 anti-CD28 antibody coated beads (Miltenyi Biotec Inc., USA), with the 
addition of either $20 \mathrm{ng} / \mathrm{ml} \mathrm{IL-6}$ or $20 \mathrm{ng} / \mathrm{ml} \mathrm{IL-23}$ or both for 5 days. The IL-17 and ROR $\gamma \mathrm{t}$ expression of Treg cells were determined by intracellular cytokine staining and flow cytometry.

\section{T cell proliferation and suppression assay}

To evaluate the suppressive function of IL-17 producing Treg cells, IL-17 producing CD4+CD25+CD161+ Treg cells or CD4+CD25+CD161+ Treg cells with rare IL-17 production was co-cultured with purified CD4+CD25- responder T cells (Tresp cells) at a 1:1 ratio and a total cell density of $1 \times 10^{5}$ cells per well in RPMI 1640 medium containing 10\% fetal bovine serum (Invitrogen, USA). Anti-CD3 antibody and anti-CD28 antibody coated beads (Miltenyi Biotec Inc., USA) as the same number as cultured cells were added to each cell-culture well to stimulate Tresp cell proliferation. As a positive control for T-cell proliferation, Tresp cells were cultured alone at $5 \times 10^{4}$ cells per well in the culture conditions described above. For the negative control, Tresp cells were cultured in the above conditions but in the absence of anti-CD3 and anti-CD28 antibody coated beads. On Day 5, Tresp cell proliferation was determined by flow cytometry.

\section{Cell sorting}

CD4+T cells were enriched from PBMCs by magnetic cell sorting (StemCell Technologies, USA). CD4+T cells were stained with PE-labeled anti-human-CD25 antibody, FITC-labeled anti-human CD161 antibody and PE-CF594-labeled anti-human-CD4 antibody (BD Biosciences), and were sorted further into T responder 
cells (Tresp, CD4+ CD25-) and two regulatory T cells subsets (CD4+CD25+CD161+ and CD4+CD25+CD161- Treg subsets) using a BD Aria II flow cytometer.

\section{Cytokine detection}

IL-6 and IL-23 in serum were measured by standard enzyme-linked immunosorbent assays (ELISA; R\&D Systems, Minneapolis, MN, USA).

\section{Clinical data and inflammation marker analysis}

All patients were assessed for complete clinical data including age, sex, disease duration, tender joint count, swollen joint count, global visual analog scale score, immunoglobulins (IgG, IgM, IgA), erythrocyte sedimentation rate (ESR), C-reactive protein (CRP), anti-keratin antibodies (AKA), anti-perinuclear factor (APF), anti-citrullinated peptide (anti-CCP) antibodies, rheumatoid factor (RF)-IgM, and RF-IgG.

The ESR was evaluated using the Westergren method. Values of $15 \mathrm{~mm} / \mathrm{h}$ for males and $20 \mathrm{~mm} / \mathrm{h}$ for females were considered normal. CRP and immunoglobulins were determined using immunonephelometry. Values $>7.9 \mathrm{mg} / \mathrm{l}$ for CRP were considered positive. Anti-CCP antibodies, RF-IgM, and RF-IgG were tested by ELISA, with normal ranges of 0-5 U/ml, 0-120 U/ml, and 0-110 U/ml, respectively. The DAS28 was calculated as previous described [19].

\section{Statistical analysis}

Data were analyzed using GraphPad Prism software, and the groups were compared using the Mann-Whitney $U$ test. The results are presented as bar graphs 
with error bars representing the means \pm SD. The potential correlation between the ratio of IL-17-producing Treg cells and clinical characteristics was examined by Spearman's rank correlation test, and $p$ values $<0.05$ indicated statistical significance.

\section{Supplementary Table}

Supplementary Table 1. Clinical and laboratory features in 12 RA patients in Figure 1.

\begin{tabular}{cc}
\hline Characteristics & Values (\%) \\
\hline Age (years) & $51.36 \pm 7.56$ \\
Men/women & $4 / 8$ \\
Disease duration (months) & $126.33 \pm 119.63$ \\
Tender joint count & $12.027 \pm 8.003$ \\
Swollen joint count & $13.273 \pm 9.119$ \\
DAS 28 & $6.018 \pm 1.595$ \\
ESR, mm/h & $60.6 \pm 34.9$ \\
CRP, mg/l & $42.5 \pm 52.4$ \\
RF-IgM positive & $10(83 \%)$ \\
RF-IgG positive & $4(33 \%)$ \\
AKA-positive & $8(67 \%)$ \\
APF-positive & $8(67 \%)$ \\
Anti-CCP-positive & $9(75 \%)$ \\
Medication & methotrexate
\end{tabular}

Clinical characteristics index were presented as mean \pm SD. DAS28: 28-joint count

Disease Activity Score; ESR: erythrocyte sedimentation rate; CRP: C-reactive protein;

RF: rheumatoid factor; AKA: anti-keratin antibodies; APF: antiperinuclear factor; Anti-CCP: anti-citrullinated peptide antibody. 


\section{Supplementary Figures}

Supplementary Figure 1. A representative graph of IL-17 producing Treg frequency in matched RA peripheral blood and synovial fluid.

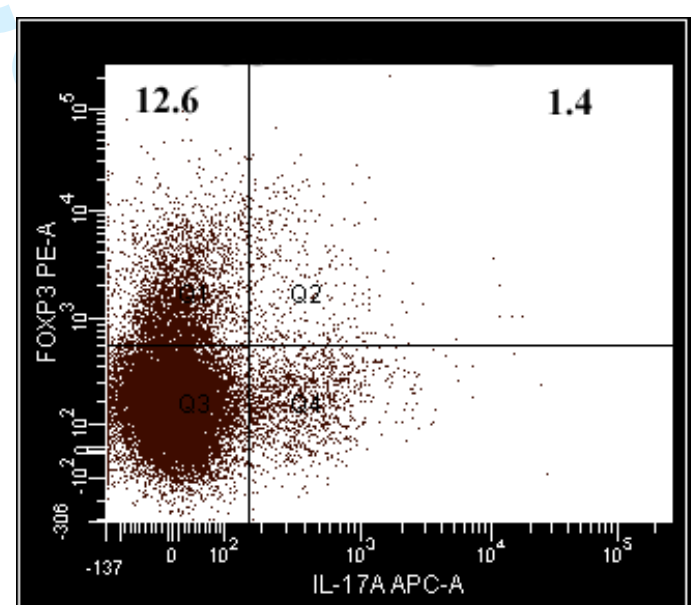

RA Peripheral Blood

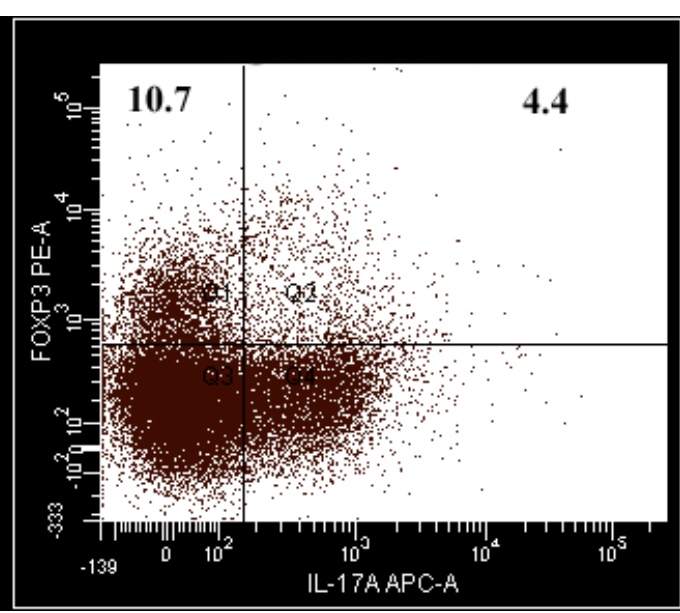

RA Synovial Fluid

Supplementary Figure 2. Correlation between IL-17 producing Treg subset from RA peripheral blood and RA serum Th17 polarizing cytokine levels
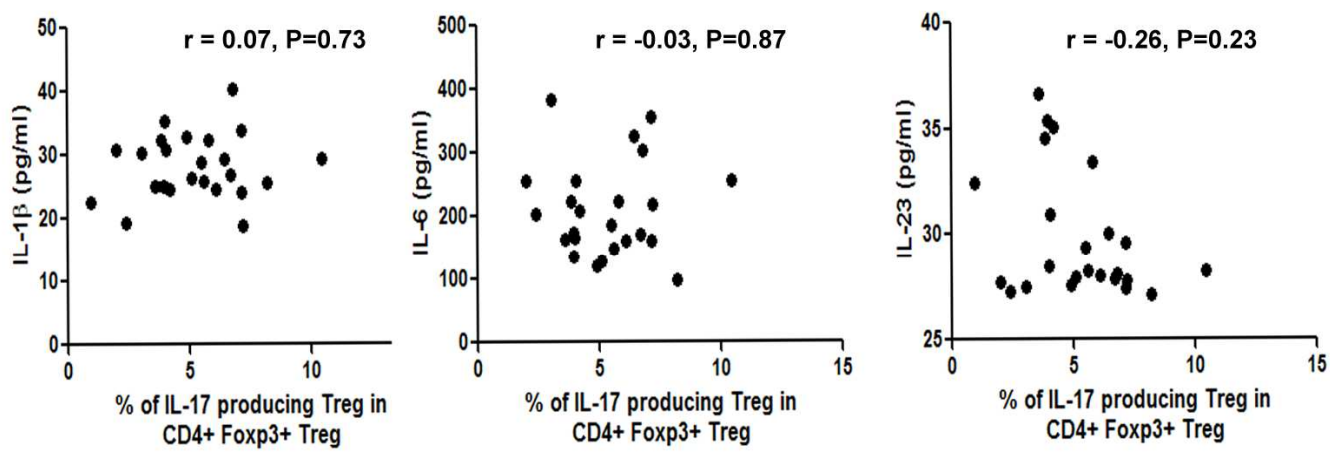